\title{
Sind wir zur Nachhaltigkeit bereit?
}

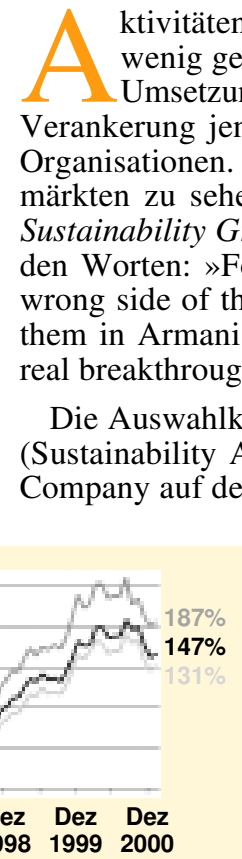

Börsenverlauf des Dow Jones Sustainablility Group Index (DJSGI, obere Kurve) im Vergleich zum Dow Jones Global Index (DJGI, unten) und dem Morgan Stanley Capital International World Index (MSCI, Mitte). Der DJSGI wurde im September 1999 eingeführt.

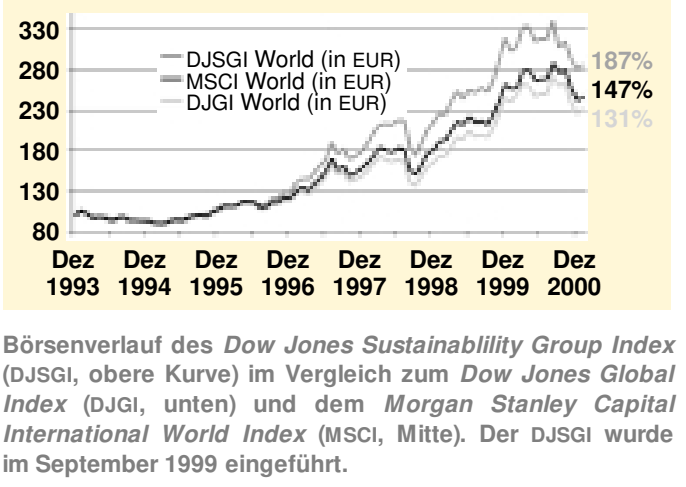
Wenn aber pragmatische Nachhaltigkeitskriterien - und davon bin ich überzeugt - zukunftsgerichtete Konzernleitungen und Strukturen identifizieren, ist mit diesem Instrument der Entwicklung von Nachhaltigkeit jedenfalls gedient.

Bereits 1965 entstand in Schweden ein Fond "Aktie Answar Myrberg" und zu Beginn der siebziger Jahre in den USA "The Pionier Fund", die sich als sozial-verantwortbare Investitionen (Social Responsible Investments, SRI) deklarierten. Viel ist in der Zwischenzeit über die Auswahlkriterien geschrieben worden. ${ }^{4}$ ) Waren die SRI erst ausschließlich auf negativen Kriterien basiert (kein Alkohol, kein Tabak, keine Waffen et cetera), werden heute zunehmend auch positive Faktoren berücksichtigt (Respekt für Umweltanliegen, gutes Verhältnis zu den Mitarbeitern, sichere Produkte et cetera). Gegenwärtig verwalten diese Fonds in den USA etwa 2160 Milliarden USD und in Europa etwa 100 Milliarden EUR.

Die Auswahlkriterien bei SRI, DJSGI wie auch anderen Unternehmensbeurteilungen oder Fonds, die auf Nachhaltigkeit zugeschnitten sind, werden im wesentlichen von kleinen Teams in kommerziellen Firmen erarbeitet. Diese leisten einen enorm wichtigen Beitrag zur Umsetzung des Nachhaltigkeitsgedankens, können aber auf sich selbst gestellt nur relativ oberflächliche, empirische Beurteilungen von Nachhaltigkeit auf der Unternehmensstufe vornehmen. Sie sind auf Unterstützung aus der Grundlagenforschung angewiesen, um ihre Konzepte und Methoden kontinuierlich weiterzuentwickeln. Gerade an dieser Unterstützung der Praxis durch akademische Forschung auf dem Gebiet der Nachhaltigkeit hapert es jedoch. Ein Schulterschluß zwischen Praxis und Wissenschaft ist eine absolute Notwendigkeit, konkrete Schritte der Wissenschaftler sind jetzt gefragt.

Damit akademische Nachhaltigkeitsforschung für die Analyse von Investitionen nützlich ist, muß sie in hohem Maße interdisziplinär (oder transdisziplinär) sein, Einschätzungen für zukünftige Entwicklungen prüfen und die gesamte Spanne von globalen Umweltproblemen bis zu Strategien individueller Firmen umfassen. Wichtige Schritte in diese Richtung wurden in den letzten Jahrzehnten von der internationalen Forschergemeinschaft unternommen. Ein erster Versuch, Rahmenbedingungen für eine nachhaltige Entwicklung aufzuzeigen, war die Studie The Limits to Growth durch den Club of Rome unter der Federführung der kürzlich 
verstorbenen Donella Meadows und ihres damaligen Ehemanns Dennis. Dieser Bericht hat eine Wandlung in der akademischen Umweltforschung eingeleitet, weg von der isolierten, fachdisziplinären Untersuchung einzelner Symptome und hin zu einer mehr integrierten Betrachtung von Nachhaltigkeitsoptionen. Die Umweltforschung an der ETH Zürich (welche die wegweisende Rolle von Donella Meadows mit einem Ehrendoktorat gewürdigt hat) und einigen anderen akademischen Institutionen ist heute exemplarisch dafür. International gibt es eine Reihe von Programmen, etwa das International Human Dimensions Programme on Global Environmental Change ${ }^{5}$, die sozial- und naturwissenschaftliche Aspekte globaler Umweltveränderungen untersuchen. Und das Konzept der Nachhaltigkeitsforschung löst allmählich das engere Konzept der Umweltforschung ab, wie besonders ein Bericht des US-amerikanischen National Research Council schon im Titel Our Common Journey: A Transition towards Sustainability deutlich macht, oder die Idee der 2000Watt-Gesellschaft im ETH-Bereich.

Dies sind Fortschritte auf dem Weg zu einer Grundlagenforschung im Gebiet der Nachhaltigkeit, die brauchbare Instrumente für Praktiker in der Finanzindustrie und ebenso in anderen Sektoren liefern könnte. Bis jetzt ist aber der wirkliche Anschluß an die Praxis noch nicht gelungen. Das liegt zum einen daran, daß die Forschung meist auf dem aggregierten Niveau von globalen Problemen oder nationalen Nachhaltigkeitsbeurteilungen (wie etwa im Fall des kürzlich auf dem World Economic Forum lancierten 2001 Environmental Sustainability Index ${ }^{6)}$ ) bleibt und selten die Brücke zur Unternehmensebene schlägt. Zum anderen ist die Vielzahl fragmentierter Nachhaltigkeitsprojekte für Praktiker verwirrend und somit schwer zugänglich, "Informationsbroker" müssen oft erst die Forschungsergebnisse zusammenfassen und transparent übermitteln. Nur wenn diese hindernden Umstände schwinden, wird es einen wirklichen Durchbruch im Nutzen der Nachhaltigkeitsforschung für die Gesellschaft geben.

Im Kielwasser der Fonds, Indices und Investmentgesellschaften haben sich im Bereich der Nachhaltigkeit bereits auch einige Fonds für Wagniskapital (private equity) gebildet. Nach eigenen Schätzungen liegen in den USA etwa 150 Milliarden USD und in Europa 2 Milliarden EUR bereit. Der Umfang des Wagniskapitals, auch im Bereich Nachhaltigkeit, nimmt stetig zu.

Das Argument (oder ist es eine Ausrede?), Nachhaltigkeit sei ausschließlich ein Kostenfaktor, ist sicherlich teilweise durch obige Informationen widerlegt. Die Wissenschaft ist jetzt gefordert, ihre Arbeit und Erkenntnisse verstärkt in den Dienst der Nachhaltigkeit zu stellen. Sind wir geistig bereit dazu? Einige von uns ja, viele aber nur "im Prinzip". Dies färbt auch auf die politische Agenda ab, die sich, wenn überhaupt, in vielen Ländern vorerst bloß mit konzeptionellen Überlegungen beschäftigt und auf Vorschläge für punktuelle Verbesserungen beschränkt. Die theoretischen und praktischen Randbedingungen zur Umsetzung der Nachhaltigkeitsidee haben sich in den letzten Jahren dermaßen verbessert, daß es keine Entschuldigung mehr geben kann für ihr weiteres Hinauszögern.

Alexander J.B. Zehnder

Direktor der Eidgenössischen Anstalt für Wasserversorgung, Abwasserreinigung und Gewässerschutz (EAWAG), Dübendorf

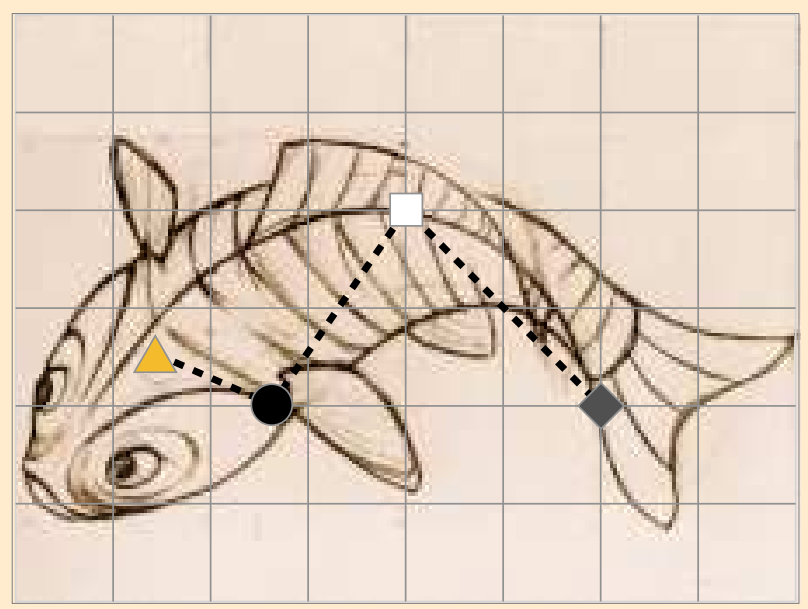

Biomonitoring mit Bioindikatoren mit Biomarkern: daraus läßt sich ein 'Naherkundungssystem' entwickeln, das Veränderungen in Ökosystemen und Lebensräumen von innen heraus anzeigt. Ein ausgezeichnetes Beispiel ist das in der Schweiz aufgebaute 'Fischnetz', es verknüpft Umweltforschung und -politik.

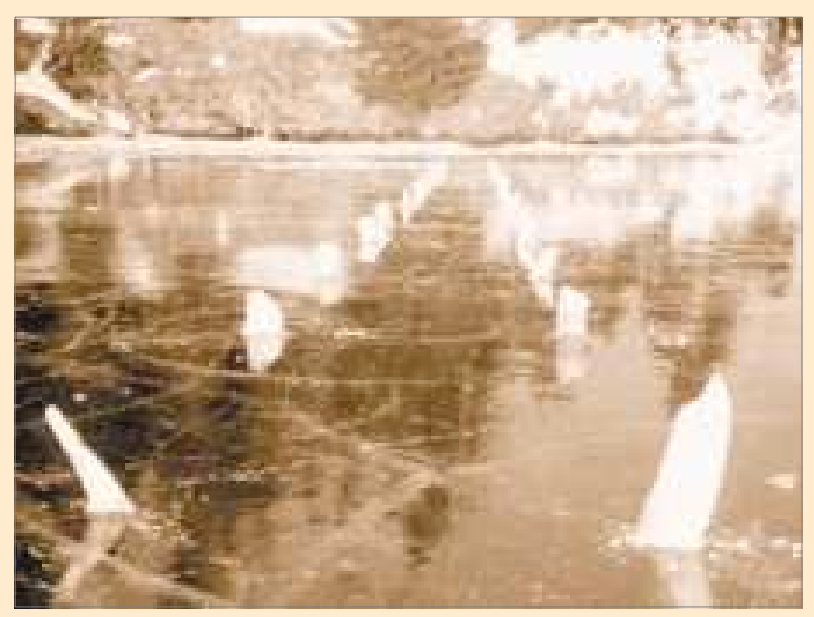

Die ökonomischen, ökologischen und sozialen Aspekte der nachhaltigen Entwicklung sind undenkbar ohne Kultur. Merkwürdigerweise nimmt jedoch die heutige Formulierung des Leitbildes Nachhaltigkeit darauf kaum Bezug.

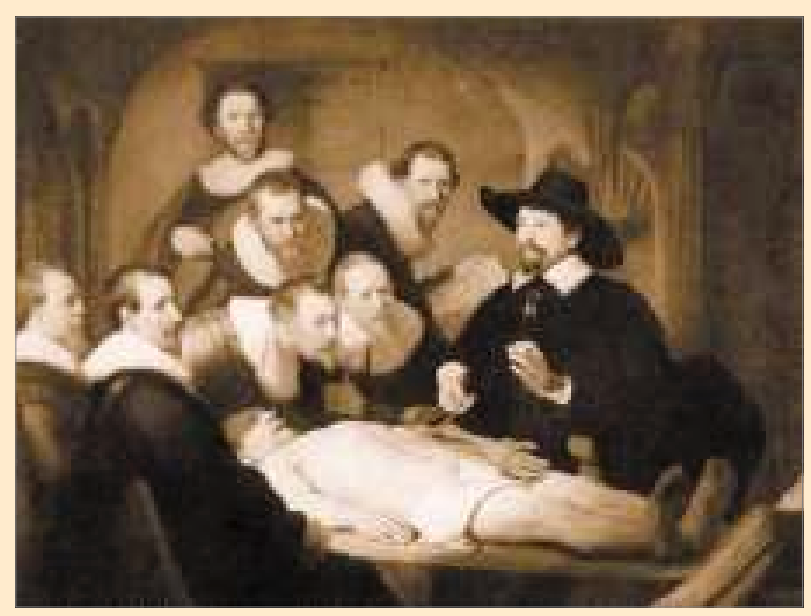

Eine Medizin, die den Patienten als bloßes Objekt betrachtet, wird diesem nicht gerecht. Ärztliches Wissen entsteht in einem Prozeß, zu dem Arzt und Patient gemeinsam beitragen. Könnte dies auch ein neues Paradigma für das Verhältnis zwischen Naturwissenschaft(en) und Natur sein? 